\title{
COMPRESSIVE MULTICHANNEL CORTICAL SIGNAL RECORDING
}

\author{
Mahdad Hosseini Kamal, Mahsa Shoaran, Yusuf Leblebici, Alexandre Schmid, Pierre Vandergheynst \\ Ecole Polytechnique Fédérale de Lausanne (EPFL), Switzerland \\ E-mail: \{mahdad.hosseinikamal, mahsa.shoaran, yusuf.leblebici, alexandre.schmid, pierre.vandergheynst\}@epfl.ch
}

\begin{abstract}
This paper presents a novel approach to acquire multichannel wireless intracranial neural data based on a compressive sensing scheme. The designed circuits are extremely compact and low-power which confirms the relevance of the proposed approach for multichannel high-density neural interfaces. The proposed compression model enables the acquisition system to record from a large number of channels by reducing the transmission power per channel. Our main contributions are the twofold. First, a CMOS compressive sensing system to realize multichannel intracranial neural recording is described. Second, we explain a joint sparse decoding algorithm to recover the multichannel neural data. The idea has been implemented at system as well as circuit levels. The simulation results reveal that the multichannel intracranial neural data can be acquired by compression ratios as high as four.
\end{abstract}

Index Terms - Sparsity, Compressive Sensing, Multichannel Signal Processing, iEEG.

\section{INTRODUCTION}

The high data rate of wireless implantable neural recording systems results in unacceptable transmission power. The amount of generated heat which can be tolerated in an implantable device, is strictly limited by the safety concern of patients. Therefore, one needs to tackle the data rate problem using a low power compression technique. Compressive sensing is a popular compression method that has superiority over traditional compression schemes because of the low power consumption and complexity of the encoder in addition to the universality with respect to the signal model. Compressive sensing samples a high dimensional signal through smaller number of linear measurements than dictated by Nyquist sampling theorem. The intracranial EEG (iEEG) signals, which are recorded through electrodes placed on the exposed surface of the cortex, are highly important in applications such as seizure detection for epilepsy monitoring. Compressive sensing has been studied in the context of biological signals such as action potentials, EEG and ECG [1, 2, 3]. The iEEG signals provide higher spatial resolution and diagnostic information compared to scalp EEG. Compressive sensing can potentially satisfy the low complexity and low power consumption requirement of the implantable neural recording systems and significantly impact the design of future implantable iEEG signal recorders.

Considering a single channel neural data, the simplest compressive sensing scheme can be realized by taking few linear measurements of the signal in a defined time frame $[1,3]$. The concept can be implemented either in the analog domain prior to digitization or downstream the ADC. The multiplication by measurement matrix and integration are performed in parallel paths which are loaded by the

The authors acknowledge the support of the Swiss NSF under grant numbers 200021-125651 and 200021-130166. rows of the measurement matrix and have a separate mixer and integrator per path. This multipath structure results in a large circuit area per channel. Therefore, the complexity of the single channel compressive sensing disqualifies the approach when we deal with a multichannel recording interface as this strategy includes large circuits in a limited die area.

To tackle the complexity and area inefficiency of the single channel compressive sensing, we propose a new measurement scheme for multichannel neural data along with a multichannel compressive recovery scheme to exploit the inter- and intra-dependencies in the multichannel neural data. As opposed to the single channel measurement scheme which needs to have access to the whole data to acquire measurements, in the proposed measurement model, as a significant advantage, the compressive measurements are acquired in real-time. The spatial sparsity of the signals generated by different channels on the electrode array is efficiently exploited in the proposed scheme.

\section{COMPRESSIVE SAMPLING}

Consider a signal $x \in \mathbb{R}^{n}$ and an orthogonal basis $\Psi \in \mathbb{R}^{n \times n}$ in which $x=\Psi \alpha$ can be sparsely represented. A signal is said to be sparse in a basis if it can be represented by $k$ non-zero coefficients, i.e. $k \ll n$.

The Compressive Sensing $[4,5,6]$ methodology employs the sparsity condition to reconstruct signals from low-rate linear compressive measurements. The linear measurements of the signal are acquired by the projection of the signal onto a small set of random vectors. The measurement vector $y \in \mathbb{R}^{m}, m \ll n$, is defined as

$$
y=\mathcal{A}(x)+z
$$

where $\mathcal{A}: \mathbb{R}^{n} \rightarrow \mathbb{R}^{m}$ is the linear map which acquires the compressive measurements and $z$ is the measurement noise. We can write the linear map in matrix form, i.e. $\mathcal{A}(x)=\mathbf{A} x$ where $\mathbf{A} \in \mathbb{R}^{m \times n}$. An approach to recover $x$ from noisy measurements vector $y$ is the socalled Basis Pursuit DeNoising problem (BPDN) $[5,6]$ in which the reconstruction casts into the following convex minimization problem

$$
\underset{\mathbf{x} \in \mathbb{R}^{\mathbf{n}}}{\operatorname{argmin}}\left\|\mathbf{\Psi}^{\mathbf{T}} \mathbf{x}\right\|_{1} \text { subject to }\|y-\mathbf{A} x\|_{2} \leq \epsilon,
$$

where $\epsilon$ is a bound over measurement noise. The $l_{q}$ norm of a vector $\alpha \in \mathbb{R}^{n}$ is defined as $\|\alpha\|_{q}=\left(\sum_{i=1}^{n}\left|\alpha_{i}\right|^{q}\right)^{1 / q}$.

\section{MULTICHANNEL IEEG COMPRESSIVE SAMPLING}

\subsection{Acquisition Model}

To recover a signal from its linear measurements, the compressive sensing methodology states that the measurement matrix $\mathbf{A}$ 
should satisfy the Restricted Isometry Property (RIP) [7]. The measurement matrices that realize the RIP condition are those randomly generated from a Gaussian distribution or matrices like the Bernoulli/Rademacher matrix. However, the aforementioned measurement methods are complex for practical problems. Therefore, the physical constraints should drive the structure of the measurement matrix.

In the case of multichannel neural recording, measurement considerations state that it is more efficient to consider a multichannel compression scheme rather than dealing with each channel separately. The sparsity of EEG signals in the spatial domain has been previously demonstrated using equivalent current dipole source representations [8]. Therefore, we choose a specific strategy to acquire the signal which fulfils the physical constraints.

Let $\mathbf{X} \in \mathbb{R}^{n_{1} \times n_{2}}$ represent the multichannel iEEG signal where $n_{1}$ is the dimension of signal in each channel and $n_{2}$ is the number of channels. The linear map obtains compressive measurements from the multichannel iEEG signal by reshaping the iEEG signal $X$ into $\hat{X} \in \mathbb{R}^{n_{2} \times n_{1}}$ and acquiring $q=p / n_{1}$ measurements from each column of $\hat{X}$. Therefore, the multichannel linear map can be represented in matrix form $\mathbf{A}_{\mathrm{M}} \in \mathbb{R}^{\left(q n_{1}\right) \times\left(n_{1} n_{2}\right)}$ as follows

$$
\mathbf{A}_{\mathrm{M}}=\left[\begin{array}{ccccc}
\boldsymbol{\Gamma}_{1} & 0 & 0 & \cdots & 0 \\
0 & \boldsymbol{\Gamma}_{2} & 0 & \cdots & 0 \\
\vdots & \vdots & \ddots & \vdots & \vdots \\
0 & 0 & 0 & \cdots & \boldsymbol{\Gamma}_{n_{1}}
\end{array}\right], \boldsymbol{\Gamma}_{i}=\left[\begin{array}{ccc}
\gamma_{1,1} & \cdots & \gamma_{1, n_{2}} \\
\gamma_{2,1} & \cdots & \gamma_{2, n_{2}} \\
\vdots & \ddots & \vdots \\
\gamma_{q, 1} & \cdots & \gamma_{q, n_{2}}
\end{array}\right]
$$

where $\boldsymbol{\Gamma}_{i} \in \mathbb{R}^{q \times n_{2}}$ and $\gamma_{i j} \in\{0,1\}$ to approximate a measurement matrix similar to the Bernoulli/Rademacher matrix. The multichannel measurement vector $y \in \mathbb{R}^{p}$ is defined as

$$
y=\mathbf{A}_{\mathrm{M}} \operatorname{vec}(\mathbf{X})+z,
$$

where $\mathbf{A}_{\mathrm{M}}$ is the multichannel measurement matrix and $\operatorname{vec}(\mathbf{X})$ represents the multichannel iEEG signal in vector form $(\operatorname{vec}(\mathbf{X}) \in$ $\left.\mathbb{R}^{n_{1} n_{2}}\right)$.

\subsection{Recovery Scheme}

Wideband neural signals consist of high amplitude spikes followed by a long period of low activity. As a consequence, the neural signals have a sparse structure in time domain. The lower frequency EEG signals have a sparse representation in Gabor or wavelet domains [1]. The multichannel neural signals have high inter-channel dependencies, as the signals recorded by the adjacent channels, depending on the spatial resolution and pitch of the electrodes, are delayed or scaled version of each other. Therefore, it is important to consider a model in order to employ the cross-correlations in the multichannel iEEG signals to efficiently reconstruct the underlying neural data. In order to recover the multichannel iEEG signal from the compressive measurements, we need to employ a sparsity domain $\boldsymbol{\Omega} \in$ $\mathbb{R}^{\left(n_{1} n_{2}\right) \times\left(n_{1} n_{2}\right)}$ for the multichannel iEEG signal $\mathbf{X}$. As explained, the underlying signals in channels share similar structures. Therefore, the multichannel basis for iEEG signals is a block diagonal matrix which has the sparsity domain along the diagonal. The multichannel iEEG basis is defined as

$$
\boldsymbol{\Omega}=\left[\begin{array}{ccccc}
\boldsymbol{\Psi} & 0 & 0 & \cdots & 0 \\
0 & \boldsymbol{\Psi} & 0 & \cdots & 0 \\
\vdots & \vdots & \ddots & & \vdots \\
0 & 0 & 0 & \cdots & \boldsymbol{\Psi}
\end{array}\right]
$$

where $\Psi \in \mathbb{R}^{n_{1} \times n_{1}}$ is the sparsity basis for each channel. The multichannel signal $\mathbf{X}$ can be recovered from the linear measurements by using the BPDN problem as

$$
\underset{\mathbf{X} \in \mathbb{R}^{\mathbf{n}_{\mathbf{1}} \times \mathbf{n}_{\mathbf{2}}}}{\operatorname{argmin}}\left\|\boldsymbol{\Omega}^{\mathbf{T}} \operatorname{vec}(\mathbf{X})\right\|_{1} \text { subject to }\left\|y-\mathbf{A}_{\mathbf{M}} \operatorname{vec}(\mathbf{X})\right\|_{2} \leq \epsilon .
$$

\section{MICROELECTRONIC ARCHITECTURE}

The system architecture of the proposed spatial compression scheme to realize the multichannel sampling strategy presented in the previous Section is depicted in Fig. 1.

Each channel of the recording scheme contains a low-noise amplifier (LNA) [9] for boosting the low-amplitude recorded signals in the front-end of the system. The amplified signals of the individual channels are sampled on $\mathrm{C}_{\mathrm{S}}$ and kept constant during $q$ measurements. The linearity of the track-and-hold circuit is guaranteed by using PMOS source-to-bulk connected source-followers. The sampled signal charges the holding capacitor in the first half cycle of the clock. In the second half, the holding capacitors of all channels are connected to the integrating capacitor, based on the random value controlling the in-pixel switch. Thus, the signals of all channels in the array are multiplied by the instantaneous random value and summed together on $\mathrm{C}_{\mathrm{INT}}\left(\mathrm{C}_{\mathrm{INT}} \gg \mathrm{C}_{\mathrm{H}}\right)$. The compressed voltage $\mathrm{V}_{\text {out }}(n)$ can be written as:

$$
\frac{\mathrm{C}_{\mathrm{H}} \phi_{\mathrm{R} 1}(n) V_{1}(n-1 / 2)+\ldots+\mathrm{C}_{\mathrm{H}} \phi_{\mathrm{R} n 2}(n) V_{n 2}(n-1 / 2)}{\mathrm{C}_{\mathrm{H}} \phi_{\mathrm{R} 1}(n)+\ldots+\mathrm{C}_{\mathrm{H}} \phi_{\mathrm{R} n 2}(n)+\mathrm{C}_{\mathrm{INT}}},
$$

where $V_{i}(n)$ is the tracked level of the signal originating from channel number $i$ at time $n T$, with $T$ being the period of the clock signal. $\phi_{\mathrm{Ri}}(n)$ is the level ( 1 or 0$)$ of the random sequence applied to $i t h$ channel at time $n T$ and $n_{2}$ is the number of channels.

In the proposed method, compressive samples are acquired from different locations and electrodes in the spatial domain, rather than over time. As a significant advantage, this design encodes the full array to one single data which is digitized using a single ADC. As a benefit of compressive sensing, the sampling rate of the latter $\mathrm{ADC}$ is $n_{2} / q$ times smaller than the sampling rate of the unique ADC which is required in a non-compressed but time-multiplexed topology. Thus, the cost of implementation in terms of in-pixel area and power is much less than previous topologies, including non-compressed and single channel compressed schemes. Using a differential topology (Fig. 1), the non-linearity and dc components caused by the source follower buffer circuit are partially removed. As an alternative, an active integrator can be used to perform the full array randomized integration and boost the signal level at the cost of an additional operational transconductance amplifier. The required speed of this amplifier is proportional to the size of the array which dictates the measurement number $q$. The compressed signal (Vout in Fig. 1) passes through a variable-gain amplifier (VGA) to further boost the level of the signal and drive the ADC.

\subsection{Pseudo Random Matrix Generation}

The actual implementation of compressive sensing requires an efficient generation of the measurement matrix in terms of power consumption and area overhead. In a single channel approach, each channel needs to be loaded with $m$ sequences, building the rows of the measurement matrix. Multiplication and integration in the analog domain (or summation in digital domain) is performed in $m$ paths. In the proposed spatial compression scheme on the other hand, each 


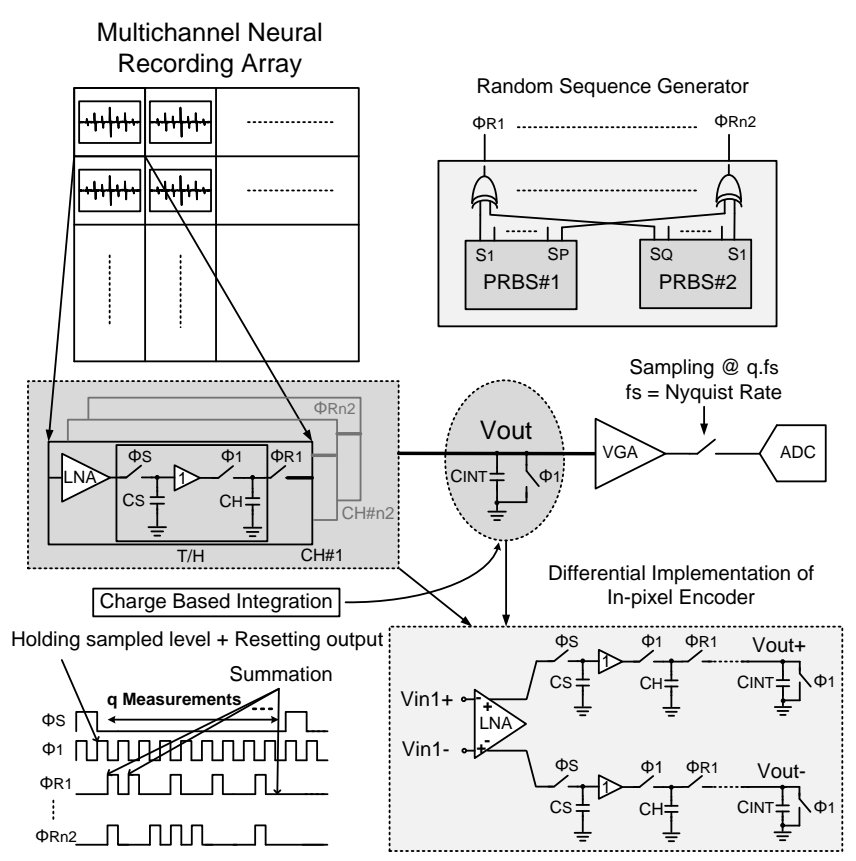

Fig. 1: Proposed multichannel compressive acquisition scheme for iEEG recording.

channel is loaded with only one sequence. The measurement matrix supporting the first $q$ measurements required for recovering the first sample of each channel is created by taking the first $q$ values of the in-channel sequences. Pseudorandom sequences which exhibit low coherence with any fixed sparsity basis [1] are a proper choice for the implementation of the measurement matrix.

In this design, the sequence generation is achieved by XORing the multiple outputs of different length Pseudo Random Bit Sequence (PRBS) generators (Fig. 1). Considering a test recording array of $4 \times 4$ and a value of $q$ equal to 4 (Compression Ratio $=16 / 4$ ), the 16 sequences driving the individual channels are generated by XORing the states of a 4-bit PRBS generator with another 5-bit PRBS generator. True Single-Phase Clocked (TSPC) flip-flops are used resulting in very low power consumption and a compact implementation. A small number of 9 flip-flops and 16 XOR gates is sufficient to generate the required sequences for 16 channels. While a single channel compression block has to be physically designed for a specific predefined $m$ and redesigned for different compression ratios, the proposed scheme is easily adaptable for different values of $q$ by simply adjusting the clock frequency. The same circuit can be used for different compression ratios and the only change is in the reconstruction code which receives $q$ as a parameter. Therefore, the proposed scheme can be perfectly tuned to find the appropriate compression ratio based on the diagnostic and medical considerations which impose the acceptable level of loss in the recovered data with respect to the original neural signal.

\section{EXPERIMENTAL RESULTS}

In order to demonstrate the effectiveness of the proposed acquisition model, multichannel intracranially recorded signals of the slices of a rat somatosensory cortex under bicuculline, which blocks the synaptic inhibition and consequently mimics epilepsy, have been used as the input. This signal includes epileptiform burst activity and extracellularly detected spikes with a maximum sampling rate of $6 \mathrm{KHz}$.

\begin{tabular}{|l|c|c|}
\hline Methods & Wavelet Transform & Time Domain \\
\hline \hline Sep-model & $17.38 \mathrm{~dB}$ & $1.2 \mathrm{~dB}$ \\
\hline Multi-model & $13.74 \mathrm{~dB}$ & $1.3 \mathrm{~dB}$ \\
\hline
\end{tabular}

Table 1: SNR comparison of two compressive neural recovery schemes for different sparsity domains.

In order to further evaluate the proposed measurement scheme, we deal with the multichannel iEEG signal with two different strategies. First, we sample each channel of the multichannel signal $x \in \mathbb{R}^{n_{1}}$ separately with a Bernoulli $\{0,1\}$ measurement matrix $\mathbf{A} \in \mathbb{R}^{m \times n_{1}}$ and the sampling ratio of $m / n_{1}=0.25$, then each channel is recovered by (2) (denoted as Sep-model). Therefore, we use a single channel basis $\Psi \in \mathbb{R}^{n_{1} \times n_{1}}$ to reconstruct the underlying neural signal. Second, we reconstruct the multichannel signal from the measurements recorded by our proposed circuit using a sampling ratio of $p /\left(n_{1} n_{2}\right)=0.25$. The multichannel signal is recovered as a whole by (6) (denoted as Multi-model). We consider the wavelet transform and time domain as two different sparsity domains for each channel. The recovery SNR of the reconstructed signal $(\hat{x})$ with respect to the original signal $(x)$ is calculated from

$$
\mathrm{SNR}=-20 \log _{10}\|x-\hat{x}\|_{2} /\|x\|_{2} .
$$

Table 1 compares the average SNR of the recovered iEEG signal for both methods. It is clear that iEEG signals are sparser in the wavelet transform than time domain, thus both recovery methods have better reconstruction quality using the wavelet transform. Moreover, the Sep-model has a better reconstruction quality than Multi-model. However, this slight improvement comes at the cost of extra power consumption and area overhead per channel for the Sep-model. The power consumption and area usage associated with the compression block in the Multi-model scheme is significantly improved, due to the real-time acquisition of measurements from full array in the spatial domain. This basically circumvents the need to place several multiplication and integration paths per channel, resulting in power and area savings while preserving acceptable reconstruction performance.

Fig. 2 demonstrates a qualitative comparison of the reconstructed neural signals using the output of the proposed circuit (Vout in Fig. 1) for five channels with respect to the original signals, when the channel sparsity domain is the wavelet transform. It is evident that the quality of spike reconstruction for Multi-model is quite good and the recovered neural signals correspond to the originals.

Finally, in order to evaluate the performance of the measurement matrix generated by the circuit, the multichannel measurement matrix is generated from a Bernoulli distribution with the same structure as the proposed measurement mechanism, with a sampling ratio of 0.25 . We use the multichannel recovery model (Multi-model) and the wavelet transform as the sparsity basis to recover the underlying multichannel signal. The recovery SNR is equal to $14.4 \mathrm{~dB}$, which reveals the accuracy of our measurement circuit.

\section{PREVIOUS WORKS}

The work presented in this paper focuses on the multichannel compression of neural signals recorded on an array of electrodes. The work by Chen [1] performs the compressive sensing in the digital domain on a single channel synthetic EEG data. Laska and Chen $([10,11])$ consider the same single channel compressive sensing approach in analog domain, prior to ADC. The method presented in this paper is shown to be significantly more efficient in terms of implementation area per channel while achieving comparable compres- 


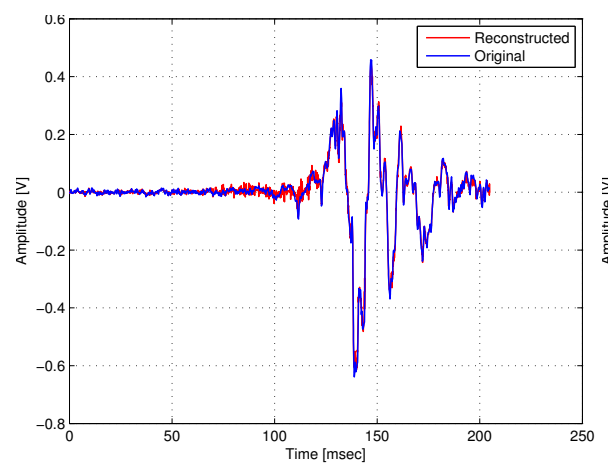

(a)

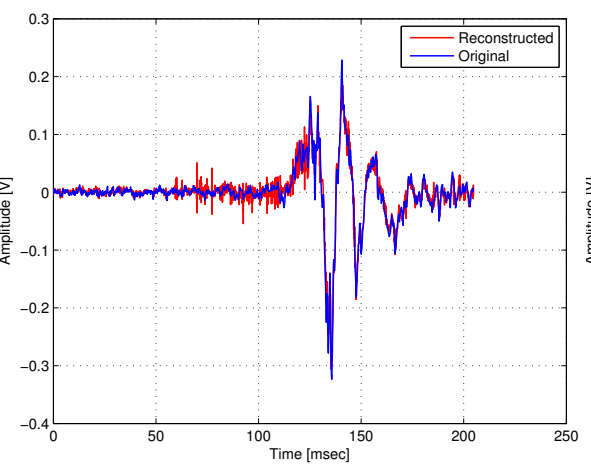

(b)

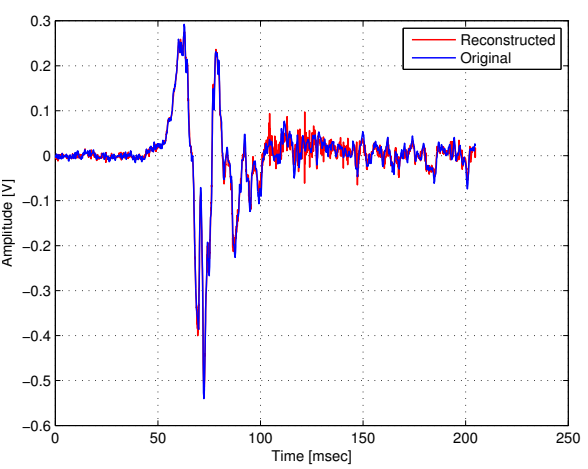

(c)

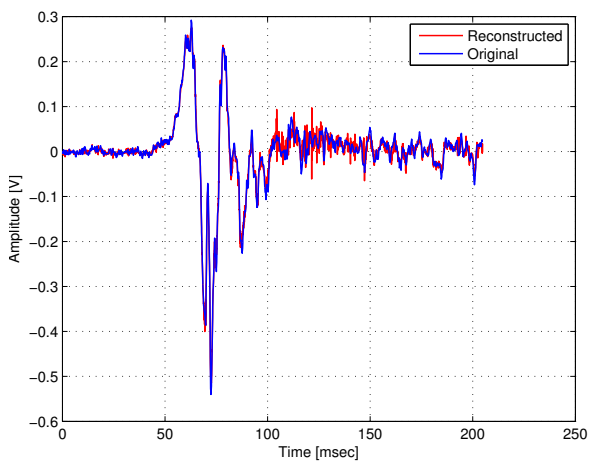

(d)

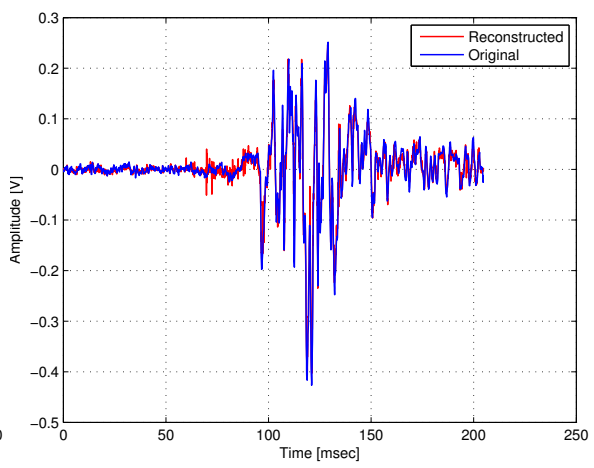

(e)

Fig. 2: Reconstruction quality of the different iEEG channels for the proposed multichannel iEEG compressive sampling scheme with sampling ratio of 0.25 . (a) Channel 1 of the multichannel iEEG, reconstruction SNR $=19.75 \mathrm{~dB}$. (b) Channel 2 of the multichannel iEEG, reconstruction SNR $=13.1 \mathrm{~dB}$. (c) Channel 3 of the multichannel iEEG, reconstruction SNR $=9.07 \mathrm{~dB}$. (d) Channel 6 of the multichannel iEEG, reconstruction SNR $=17.5 \mathrm{~dB}$. (e) Channel 15 of the multichannel iEEG, reconstruction $\mathrm{SNR}=13.59 \mathrm{~dB}$.

sion and power reduction performance to the previous circuit-level compressive sensing systems for neural recording.

\section{CONCLUSION}

This paper presents a new microelectronic system for compressive recording of multichannel high density intracranial neural signals. We developed a reconstruction model to efficiently reconstruct the multichannel neural signal based on a multichannel basis. To avoid the power and area overhead of placing an individual random sequence generator per channel, an efficient method for multi-output sequence generation is also presented. Finally, without applying any thresholding or signal-dependent preprocessing, the potential interest of our proposed scheme is evaluated through system and circuit level simulations.

\section{REFERENCES}

[1] F. Chen, A. P. Chandrakasan, and V. Stojanovic, "Design and Analysis of a Hardware-efficient Compressed Sensing Architecture for Data Compression in Wireless Sensors," IEEE J. Solid-State Circuits, pp. 744-756, 2012.

[2] H. Mamaghanian, N. Khaled, D. Atienza, and P. Vandergheynst, "Compressed Sensing for Real-time Energy-efficient ECG Compression on Wireless Body Sensor Nodes," IEEE Trans. Biomed. Eng., pp. 24562466, 2011.

[3] A. M. R. Dixon, E. G. Allstot, D. Gangopadhyay, D. J. Allstot, "Compressed Sensing System Considerations for ECG and EMG Wireless
Biosensors," IEEE Transactions on Biomedical Circuits and Systems, pp. 156-166, 2012.

[4] E. J. Candès, J. Romberg and T. Tao, "Stable Signal Recovery from Incomplete and Inaccurate Measurements," Communications on Pure and Applied Mathematics, pp. 1207-1223, 2006.

[5] E. J. Candès, "Compressive Sampling," International Congress of Mathematicians, 2006.

[6] D. Donoho, "Compressed Sensing," IEEE Transactions Information Theory, 2006.

[7] E. J. Candès and J. Romberg, "Quantitative Robust Uncertainty Principles and Optimally Sparse Decompositions," Foundation of Computational Mathematics, pp. 227-254, 2006.

[8] L. Miao, J. J. Zhang, A. Papandreou-Suppappola, C. Chakrabarti, "Neural Activity Tracking Using Spatial Compressive Particle Filtering," IEEE International Conference on Acoustics, Speech and Signal Processing, pp. 3461-3464, 2012.

[9] M. Shoaran, C. Pollo, Y. Leblebici and A. Schmid, "Design Techniques and Analysis of High-Resolution Neural Recording Systems Targeting Epilepsy Focus Localization," International Conference of the IEEE EMBS, pp. 5150-5153, 2012.

[10] J. N. Laska, S. Kirolos, M. F. Duarte, T. S. Ragheb, R. G. Baraniuk, and Y. Massoud, "Theory and Implementation of Analog-to-information Converter Using Random Demodulation," Proc. 2007 IEEE Int. Symp. Circuits and Systems (ISCAS), pp. 1959-1962, 2007.

[11] X. Chen, Z. Yu, S. Hoyos, B. M. Sadler, and J. Silva-Martinez, “A Sub-Nyquist Rate Sampling Receiver Exploiting Compressive Sensing," IEEE Trans. Circuits Syst. I, pp. 507-520, 2010. 